\title{
Subcortical anatomy as an anatomical and functional landmark in insulo-opercular gliomas: implications for surgical approach to the insular region
}

\author{
Juan Martino, MD, PhD, ${ }^{1}$ David Mato, MD, ${ }^{1}$ Enrique Marco de Lucas, MD, ${ }^{2}$ \\ Juan A. García-Porrero, MD, PhD, ${ }^{3}$ Andreu Gabarrós, MD, PhD, ${ }^{4}$ Alejandro Fernández-Coello, MD, ${ }^{4}$ \\ and Alfonso Vázquez-Barquero, MD, $\mathrm{PhD}^{1}$
}

Departments of ${ }^{1}$ Neurological Surgery and ${ }^{2}$ Radiology, Hospital Universitario Marqués de Valdecilla and Fundación Instituto de Investigación Marqués de Valdecilla (IDIVAL); ${ }^{3}$ Department of Anatomy and Cellular Biology, Cantabria University, Santander; and ${ }^{4}$ Department of Neurological Surgery, Hospital Universitari de Bellvitge, Barcelona, Spain

\begin{abstract}
OBJECT Little attention has been given to the functional challenges of the insular approach to the resection of gliomas, despite the potential damage of essential neural networks that underlie the insula. The object of this study is to analyze the subcortical anatomy of the insular region when infiltrated by gliomas, and compare it with the normal anatomy in nontumoral hemispheres.

METHODS Ten postmortem human hemispheres were dissected, with isolation of the inferior fronto-occipital fasciculus (IFOF) and the uncinate fasciculus. Probabilistic diffusion tensor imaging (DTI) tractography was used to analyze the subcortical anatomy of the insular region in 10 healthy volunteers and in 22 patients with insular Grade II and Grade III gliomas. The subcortical anatomy of the insular region in these 22 insular gliomas was compared with the normal anatomy in 20 nontumoral hemispheres.

RESULTS In tumoral hemispheres, the distances between the peri-insular sulci and the lateral surface of the IFOF and uncinate fasciculus were enlarged $(p<0.05)$. Also in tumoral hemispheres, the IFOF was identified in $10(90.9 \%)$ of 11 patients with an extent of resection less than $80 \%$, and in $4(36.4 \%)$ of 11 patients with an extent of resection equal to or greater than $80 \%$ (multivariate analysis: $p=0.03$ ).

CONCLUSIONS Insular gliomas grow in the space between the lateral surface of the IFOF and uncinate fasciculus and the insular surface, displacing and compressing the tracts medially. Moreover, these tracts may be completely infiltrated by the tumor, with a total disruption of the bundles. In the current study, the identification of the IFOF with DTI tractography was significantly associated with the extent of tumor resection. If the IFOF is not identified preoperatively, there is a high probability of achieving a resection greater than $80 \%$.

http://thejns.org/doi/abs/10.3171/2014.11.JNS141992
\end{abstract}

KEY WORDS DTI tractography; fiber dissection; glioma; inferior fronto-occipital fasciculus; insula; uncinate fasciculus; anatomy

$\mathrm{T}$ HE insula of Riel is located at the depth of the sylvian cistern, covered by the frontal, parietal, and temporal operculi. It overlies the striatum and thalamus, and it is covered by the trunk of the middle cerebral artery and its branches. ${ }^{30,39}$ Insular gliomas are frequently encountered in neurosurgical practice, as the insula appears to be a preferential location for low-grade gliomas. An epidemiological study performed by Duffau and Capelle ${ }^{9}$ showed that these lesions accounted for up to $25 \%$ of all low-grade gliomas and $10 \%$ of all high-grade gliomas.

The insular region is a highly connected area, as an intricate network of afferent and efferent projections connect it with adjacent and distant cortical regions. ${ }^{14,21,26,30}$ There are 2 prominent associative bundles that run under the in-

ABBREVIATIONS DTI = diffusion tensor imaging; FLAIR = fluid-attenuated inversion recovery; IES = intraoperative electrical stimulation; IFOF = inferior fronto-occipital fasciculus; $\mathrm{ROI}=$ region of interest.

SUBMITTED August 29, 2014. ACCEPTED November 5, 2014.

INCLUDE WHEN CITING Published online May 8, 2015; DOI: 10.3171/2014.11.JNS141992.

DISCLOSURE Juan Martino receives specific funding from the 11/18 API grant entitled "Estudio de la conectividad cerebral mediante disección de fibras structural," Fundación Instituto de Investigación Marqués de Valdecilla (IDIVAL), Santander, Cantabria, Spain. October, 8, 2010. 
sular lobe: the inferior fronto-occipital fasciculus (IFOF) and the uncinate fasciculus. These 2 tracts connect distant areas at the frontal, temporal, and occipital lobes. Other important connections in close relation to the insula are the pyramidal pathway, claustro-opercular connections, thalamocortical connections, anterior commissure, subcallosal fasciculus, and accumbofrontal fasciculus. Gliomas are characterized by a diffuse and infiltrative pattern of growth, with cellular invasion and migration along white matter fiber tracts. ${ }^{3,20,38}$ Given the important number of connections between the insula and surrounding areas, it is not surprising that insular gliomas are often extended to the adjacent lobes. Indeed, insular gliomas restricted to the insula (Yaşargil Type 3A) are rare, and the tumor frequently affects the operculi (Yaşargil Types 3B, 5A, and 5B). $8,32,44$

The resection of insular gliomas represents a challenging and controversial area in neurosurgery. Numerous publications have recently focused on the analysis of the anatomical challenges of insular approaches: the limited operative space, with risk of excessive opercular retraction; the difficulty in reaching the limen insulae, with potential to injure the lenticulostriate arteries; the challenges of approaching the posterior insula situated in the depth of the sylvian cistern; and the risk of spasm due to manipulation of the middle cerebral artery. ${ }^{8,17,19,32,34,40}$ However, less attention has been given to the functional challenges of the insular approach, despite the potential damage to essential neural networks that underlie the insula. In fact, recent publications report high rates of learning and memory impairment after insula glioma surgery. ${ }^{43}$ The aim of the present study is to analyze the subcortical anatomy of the insular region when infiltrated by gliomas, using fiber dissection and diffusion tensor imaging (DTI) tractography, and to compare it with the normal anatomy in nontumoral hemispheres. We focused this anatomical analysis on the IFOF and uncinate fasciculus, as they are the two major associative tracts that cross the insula and they have an important role in high cognitive functions such as language and memory. In light of these new anatomical data, we will review from a functional perspective the major steps of the surgical approach to insulo-opercular gliomas.

\section{Methods}

\section{Cortex-Sparing Fiber Dissection}

Ten human cerebral hemispheres (5 right and 5 left) were removed from bodies embalmed with $10 \%$ formalin solution for at least 40 days. The mean age of the donors from whom the brains were obtained was 71.5 years (range 65.5 to 78.5 years). Our group has previously described the dissection methodology, i.e., cortex-sparing fiber dissection, in detail. ${ }^{21-24,26}$ The sylvian cistern was widely split, the insular surface exposed, and the insular cortex lifted, exposing the extreme capsule. At the anteroinferior portion of the extreme and external capsules, the uncinate fasciculus and the IFOF were exposed (Fig. 1). In this region, both fasciculi run parallel, with a posterior and inferior orientation; the IFOF is located dorsal and posterior to the uncinate fasciculus.

At the periphery of the insula, both fascicles cross the cerebral isthmi. The isthmi are located between the ventricle and the peri-insular sulci, and they are neural bridges that connect the frontal, parietal, occipital, and temporal lobes to the central core (deep region of the hemisphere that contains the caudate nucleus; putamen; globus pallidus; thalamus; claustrum; fornix; and internal, external, and extreme capsules). ${ }^{30}$ The inferior isthmus is located between the inferior limiting sulcus of the insula and the temporal horn and is also referred to as the stem of the temporal lobe (temporal stem). The superior isthmus is located between the superior limiting sulcus of the insula and ventricular body and atrium. The anterior isthmus is located deep to the anterior limiting sulcus of the insula (Figs. 2-4).

Inferiorly, the IFOF and uncinate fasciculus cross the temporal isthmus. The auditory radiations and the claustro-opercular and insulo-opercular fibers of the external and extreme capsules pass through the temporal stem above the IFOF, whereas the optic radiations pass below. Superiorly, both fascicles cross the anterior and superior isthmi. The claustro-opercular and insulo-opercular fibers of the external and extreme capsules pass through the anterior and superior isthmi superficial to the IFOF (Fig. 1).

\section{Subject Population}

Patients were selected according to the following criteria: 1) age older than 18 years; 2) gliomas involving the insula; 3) histological confirmation of an infiltrative World Health Organization (WHO) Grade II glioma or anaplastic glioma; 4) primary craniotomy for tumor resection (no previous craniotomy for tumor resection); and 5) no chemotherapy or radiotherapy before the current surgery. Between July 2009 and June 2014, 15 consecutive patients who underwent surgery at the Hospital Universitario Marqués de Valdecilla (Santander, Spain), and 7 patients who underwent surgery at the Hospital Universitari de Bellvitge (Barcelona, Spain), met all inclusion criteria and were eligible for the study.

Five healthy volunteers were also included in this study, and their subcortical anatomy of the insular region was studied with DTI tractography. The volunteers' mean age was 30.9 years old (range 19.5-42.5 years).

All participants gave their informed consent to participate in the research; all procedures were approved by the Hospital Universitario Marqués de Valdecilla Committee of Human Research, and all research was conducted according to the Declaration of Helsinki.

\section{MRI and DTI Tractography Analysis}

All subjects were studied using brain MRI performed on a whole-body 3.0-T scanner (Achieva 3.0T; Philips Healthcare) with an 8-channel head coil. MRI was performed 1 month before surgery and 3 months after surgery. The imaging protocol included axial, sagittal, and coronal T1-weighted images (simple and contrast enhanced), T2weighted images, and fluid-attenuated inversion recovery (FLAIR) images. In the tumoral hemispheres, manual segmentation was performed with region-of-interest (ROI) analysis to measure tumor volumes $\left(\mathrm{cm}^{3}\right)$ on the ba- 

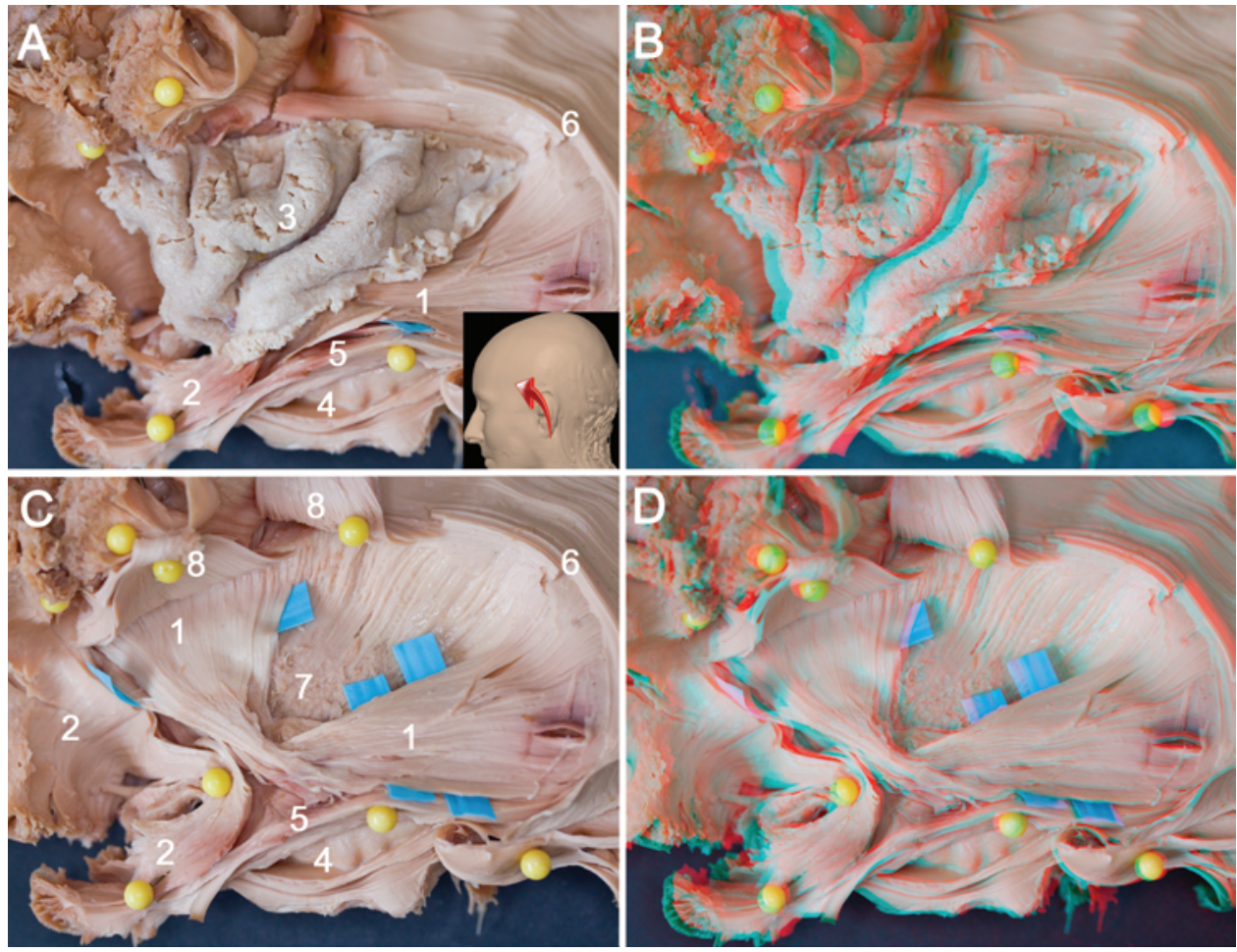

FIG. 1. Photographs of cadaveric dissection illustrating cortex-sparing fiber dissection. A: Fiber dissection of the tracts that pass through the insular region in the left hemisphere. The temporal and parietal operculi have been removed to expose the insula surface (3) and the tracts that enter the insula. B: Two photographs of the dissection superimposed to provide a 3D perspective. C: The insula, claustrum, and external and extreme capsules have been removed to expose the deep bundles that run under the insula: the IFOF (1) and the uncinate fasciculus (2). Small pieces of blue paper have been placed between the IFOF and deeper structures to demonstrate that the fibers of this bundle were completely dissected and isolated from the surrounding fiber tracts. D: 3D illustration of the previous dissection. $1=$ inferior fronto-occipital fasciculus; $2=$ uncinate fasciculus; $3=$ insula; $4=$ temporal horn of the ventricle; $5=$ optic radiations; $6=$ arcuate fasciculus; $7=$ putamen; $8=$ claustro- and insulo-opercular connections. Figure is available in color online only.

sis of FLAIR axial slices. ${ }^{36}$ The extent of tumor resection was determined by comparing MRI scans obtained before surgery with those obtained 3 months after surgery. The extent of resection was calculated as (preoperative tumor volume - postoperative tumor volume) / preoperative tumor volume.

In accordance with methodology previously described by our group, ${ }^{23}$ DTI was performed using a single-shot, multislice, spin echo-echo planar sequence with the following attributes: diffusion sensitization $1300 \mathrm{sec} / \mathrm{mm}^{2}$, TR $9577 \mathrm{msec}$, TE $77 \mathrm{msec}$, voxel size $2 \mathrm{~mm}^{3}$, no gap between slices, matrix $224 \times 224$. Sixty-four diffusion gradient directions were obtained. The DTI data sets and anatomical MRI scans were analyzed with Fiber Track software from MR Workspace (Philips Healthcare) for diffusion-tensor analysis and fiber tracking. To increase the number of detectable fibers, we applied a probabilistic tracking algorithm with progressive lowering of the fractional anisotropy value. A knowledge-based multiple ROI approach was applied, in which the tracking algorithm was initiated from user-defined seed regions. Axonal projections were traced both in anterograde and retrograde directions according to the direction of the principal eigenvector in each voxel of the region of interest.

\section{Surgical Technique}

All patients underwent surgery with direct cortical and subcortical electrical stimulation (19 patients with an asleep-awake-asleep technique and 3 patients under general anesthesia), using a methodology previously described by our group. ${ }^{22,25}$ Briefly, a bipolar electrode with a 5-mm space between the tips and delivering a biphasic current (square-wave pulses in 4-second trains at $60 \mathrm{~Hz}$, single pulse phase duration $1 \mathrm{msec}$, and amplitude 2-8 mA) (Nimbus; Hemodia) was applied to the brain. Sensorimotor mapping was performed first to identify the primary motor and sensory areas. The patient was then asked to count from 1 to 50 , over and over, and to perform picture naming using the DO80 test, which consists of 80 black and white pictures selected according to variables such as frequency, familiarity, age of acquisition, and education level. ${ }^{27}$ During tumor removal, direct stimulation at the subcortical level was applied to identify the deep functional limits of resection, i.e., white matter pathways. A positive language site was associated with a patient's inability to count or name objects during $66 \%$ of the stimulation trials. Functional areas identified were located in preoperative DTI tractography images using a neuronavigation system. Resection proceeded until eloquent struc- 

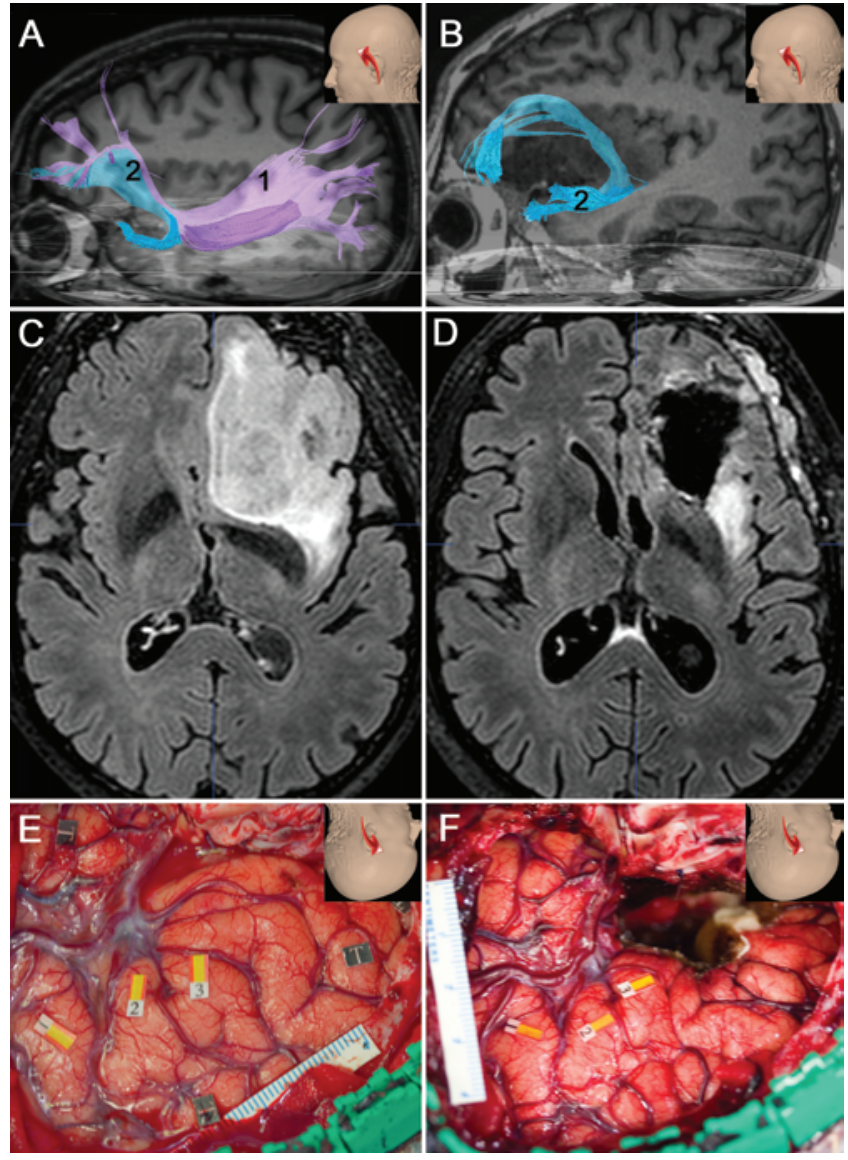

FIG. 2. A: Sagittal T1-weighted MR images with DTI tractography reconstruction of the tracts passing through the left insula in a healthy volunteer. 1 = inferior fronto-occipital fasciculus (IFOF); 2 = uncinate fasciculus. B-D: MR images obtained in a patient with a left frontotemporoinsular anaplastic astrocytoma (Yaşargil Type 5A; Berger-Sanai Classes I, III, and IV). B: Preoperative sagittal T1-weighted MRI images with DTI tractography reconstruction of the tracts passing through the insula. The uncinate fasciculus (2) had an abnormal trajectory as it crossed the superior isthmus but not the anterior isthmus. Interestingly, it was not possible to reconstruct the IFOF, probably due to the tumor infiltration. C: Preoperative axial FLAIR-weighted MRI image. The preoperative tumor volume was $79.8 \mathrm{~cm}^{3}$. D: Postoperative axial FLAIR-weighted MRI image, which demonstrates that the resection was extended to the deep tumor margin at the lateral surface of the striatum. The postoperative tumor volume was $10.8 \mathrm{~cm}^{3}$, and the extent of resection was $86.5 \%$. E: Intraoperative photograph taken before tumor resection, where the frontal and temporal lobes and the sylvian fissure are exposed. Intraoperative electrical stimulation of the cortex elicited the following responses: flag with number $1=$ speech arrest at the ventral premotor cortex, flag with number $2=$ anomia at the pars opercularis of the inferior frontal gyrus, flag with number $3=$ semantic paraphasia at the pars triangularis of the inferior frontal gyrus. F: Intraoperative photograph taken after tumor resection. Subcortical electrical stimulation failed to identify eloquent structures at the margins of the surgical cavity. Figure is available in color online only.

tures were encountered around the surgical cavity. Brain shift is the displacement of the brain that occurs during surgery because of the effect of gravity and tumor resection, and displacements of as much as $2.4 \mathrm{~cm}$ have been reported. ${ }^{29}$ This error, however, did not bias the correlation between preoperative DTI tractography and the extent of tumor resection.

\section{Statistical Analysis}

Frequency distributions and summary statistics were calculated for all variables; values are expressed as mean and range. A Kolmogorov-Smirnov test was used to study the distribution of each variable and probability and quantile plots were used to confirm it. The majority did not follow a normal distribution, and nonparametric tests were used for comparisons. The 22 hemispheres with insular tumors were compared with the 20 hemispheres without insular tumors (10 hemispheres dissected and 10 hemispheres of healthy volunteers studied with DTI tractography). The Mann-Whitney U-test was used to determine the relationship between the independent variable (hemispheres with and without tumor) and quantitative variables (distances measured). The Fisher exact test was used to determine the relationship between the independent variable (hemispheres with and without tumor) and qualitative variables (identification of each tract related to the insula). In the tumoral hemispheres, the identification of each tract with DTI tractography was tested for significant association with the extent of resection with a logistic regression multivariate analysis adjusted for age, tumor side, tumor location, histology, and preoperative tumor volume. A significance level of $5 \%(\mathrm{p}<0.05)$ was accepted in all cases. SPSS software, version 19.0 (SPSS, UK), was used for the statistical analysis.

\section{Results}

\section{Patient Population}

The demographic, radiological, and pathological characteristics of the subjects and of their tumors are listed in Table 1.

The patient population with insular tumors included 11 men and 11 women, with a mean age of 43.6 years (range 22.1-66.4 years). Sixteen patients (72.7\%) presented with seizures of recent onset. All patients had an intact preoperative neurological examination. The median preoperative Karnofsky Performance Status score was 90 (range 90-100). The mean preoperative tumor volume was 85.9 $\mathrm{cm}^{3}$ (range $25.5-173.8 \mathrm{~cm}^{3}$ ), the mean postoperative tumor volume was $17.7 \mathrm{~cm}^{3}$ (range $4.9-49.8 \mathrm{~cm}^{3}$ ), and the mean extent of resection was $78.1 \%$ (range $46.4 \%-96.1 \%$ ).

One week after surgery, new onset of language deficit was present in 12 patients: mild dysphasia in 7 cases and moderate dysphasia in 5 cases. One patient developed Foix-Chavany-Marie syndrome with anarthria and bilateral facial weakness, with complete recovery 8 days after surgery. Postoperative motor deficits were not reported. Three months after surgery, all patients except 2 had complete resolution of the deficits. Two patients had a permanent mild dysphasia.

\section{Tracts Identified at the Insular Region}

The tracts identified and distances measured in each hemisphere are listed in Tables 2 and 3.

The IFOF was identified in all the nontumoral hemispheres and in 14 tumoral hemispheres (63.6\%). This difference was statistically significant $(\mathrm{p}=0.004)$. The IFOF crossed the superior isthmus in all nontumoral hemispheres $(100 \%)$ and in $8(57.1 \%)$ of 14 tumoral hemi- 


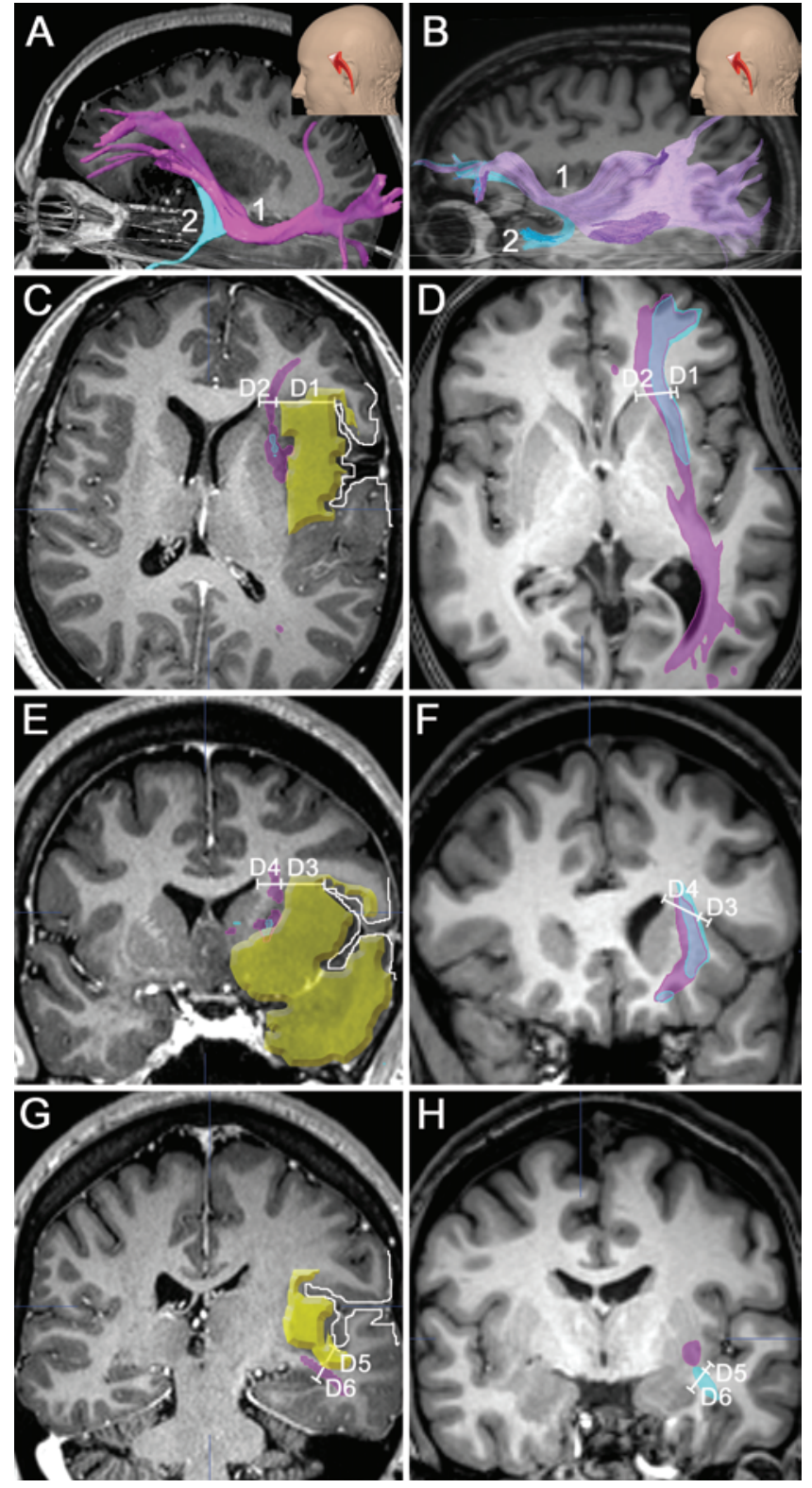

spheres. This difference was statistically significant $(\mathrm{p}=$ 0.002 ). The IFOF crossed the anterior and inferior isthmi in all the nontumoral and tumoral hemispheres.

In tumoral hemispheres, the identification of the IFOF with DTI tractography was significantly associated to the extent of tumor resection. The IFOF was identified in $10(90.9 \%)$ of 11 patients with an extent of resection less than $80 \%$, and in $4(36.4 \%)$ of 11 patients with an extent of resection equal to or greater than $80 \%$. Using univariate analysis, the difference observed between these groups was statistically significant $(\mathrm{p}=0.008)$. Using a multivariate logistic regression model adjusted by age, tumor side, tumor location, histology, and preoperative tumor volume, the difference was also statistically significant $(p=0.03)$.

The uncinate fasciculus was identified in all the nontumoral hemispheres and in 13 tumoral hemispheres (59.1\%). This difference was statistically significant $(\mathrm{p}=$
FIG. 3. A, C, E, and G: T1-weighted MRI images with DTI tractography reconstruction of the tracts passing through the left insula in a patient with a left frontotemporoinsular WHO Grade II astrocytoma (Yaşargil Type 5B; Berger-Sanai Classes I, II, III, and IV). B, D, F, and H: T1weighted MRI images with DTI tractography reconstruction of the tracts passing through the left insula in a healthy volunteer. A: Preoperative sagittal T1-weighted MRI images with DTI tractography reconstruction of the tracts passing through the insula in the frontotemporoinsular glioma. 1 = Inferior fronto-occipital fasciculus; 2 = uncinate fasciculus. B: Sagittal T1-weighted MRI images with DTI tractography reconstruction of the tracts passing through the insula in the healthy volunteer. 1 = Inferior fronto-occipital fasciculus; 2 = uncinate fasciculus. C: Preoperative axial T1-weighted MRI images with DTI tractography reconstruction of the tracts passing through the anterior isthmus in the frontotemporoinsular glioma. The tracts are displaced medially at the anterior isthmus due to tumor compression. The deep surface of the bundles are in direct contact with the lateral surface of the putamen. Inferior fronto-occipital fasciculus (purple); uncinate fasciculus (blue). Please refer to the text for definitions of distance measures. D: Axial T1weighted MRI images with DTI tractography reconstruction of the tracts passing through the anterior isthmus in the healthy volunteer. E: Preoperative coronal T1-weighted MRI images with DTI tractography reconstruction of the tracts passing through the superior isthmus in the frontotemporoinsular glioma. Inferior fronto-occipital fasciculus (purple); uncinate fasciculus (blue). F: Coronal T1-weighted MRI images with DTI tractography reconstruction of the tracts passing through the superior isthmus in the healthy volunteer. G: Preoperative coronal T1weighted MRI images with DTI tractography reconstruction of the tracts passing through the temporal stem in the frontotemporoinsular glioma. Inferior fronto-occipital fasciculus (purple); uncinate fasciculus (blue). $\mathrm{H}$ : Axial T1-weighted MRI images with DTI tractography reconstruction of the tracts passing through the temporal stem in the healthy volunteer. Figure is available in color online only.

$0.001)$. The uncinate fasciculus crossed the superior isthmus in $8(40 \%)$ of 20 nontumoral hemispheres and in 4 (30.8\%) of 13 tumoral hemispheres. This difference was not statistically significant $(\mathrm{p}>0.05)$. The uncinate fasciculus crossed the anterior isthmus in all the nontumoral hemispheres $(100 \%)$ and in $11(84.6 \%)$ of 13 tumoral hemispheres. This difference was not statistically significant $(\mathrm{p}>0.05)$. The uncinate fasciculus crossed the inferior isthmus in all the nontumoral and tumoral hemispheres.

The optic radiations and pyramidal pathway were identified in all the nontumoral and tumoral hemispheres. Postoperative DTI tractography was available in 15 cases: 11 cases in which the IFOF was identified preoperatively and in 4 cases in which the IFOF was not identified preoperatively. Postoperative tractography revealed that the IFOF was not identified in any of the cases in which this tract was not reconstructed preoperatively, and it was identified in $9(81.8 \%)$ of 11 cases in which this tract was reconstructed preoperatively.

\section{Distances Measured}

The following distances were measured at the insular region (Figs. 3 and 4 and Table 3):

- D1: distance measured at the anterior isthmus, the shortest lateromedial distance between the anterior limiting sulcus of the insula and the lateral surface of the IFOF and uncinate fasciculus.

- D2: distance measured at the anterior isthmus, the 


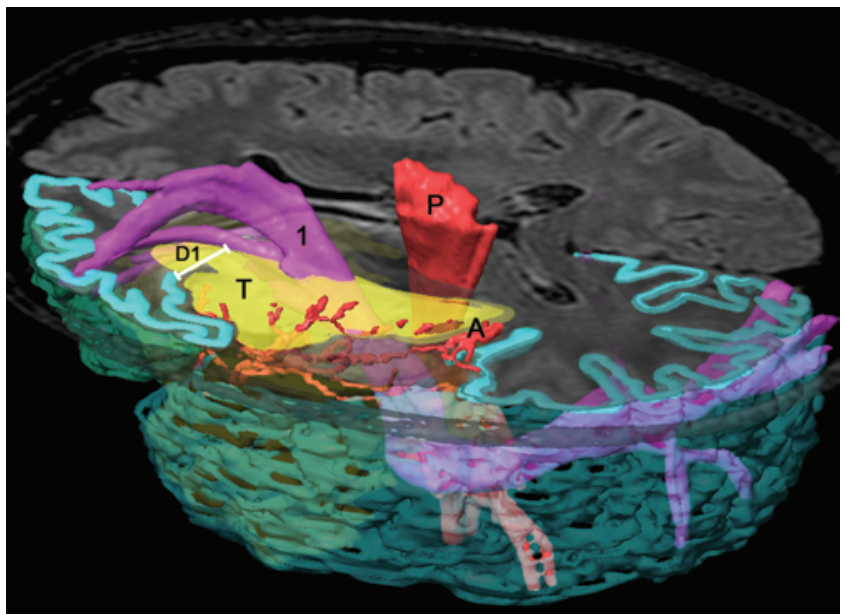

FIG. 4. 3D illustration of the tracts passing through the left insula of the same case presented in Fig. 3. 1 = inferior fronto-occipital fasciculus; $\mathrm{A}=$ middle cerebral artery; D1 = the shortest lateromedial distance between the anterior limiting sulcus of the insula and the lateral surface of the IFOF and uncinate fasciculus; $P=$ pyramidal pathway; $T=$ tumor. Figure is available in color online only.

shortest lateromedial distance between the ventricle and the lateral surface of the IFOF and uncinate fasciculus.

- D3: distance measured at the superior isthmus, the shortest lateromedial distance between the superior limiting sulcus of the insula and the lateral surface of the IFOF and uncinate fasciculus.

- D4: distance measured at the superior isthmus, the shortest lateromedial distance between the ventricle and the lateral surface of the IFOF and uncinate fasciculus.

- D5: distance measured at the temporal isthmus, the shortest superoinferior distance between the inferior limiting sulcus of the insula and the superior surface of the IFOF and uncinate fasciculus.

- D6: distance measured at the temporal isthmus, the shortest superoinferior distance between the ventricle and the superior surface of the IFOF and uncinate fasciculus.

- D7: the shortest lateromedial distance between the limen insulae and the lateral surface of the IFOF and uncinate fasciculus.

- D8: the shortest lateromedial distance between the lateral margin of the anterior perforated substance and the lateral surface of the IFOF and uncinate fasciculus.

- D9: the shortest lateromedial distance between the insular apex and the lateral surface of the IFOF and uncinate fasciculus.

- D10: the shortest lateromedial distance between the lateral surface of the putamen and the medial surface of the IFOF and uncinate fasciculus.

- D11: distance measured at the superior isthmus, the shortest lateromedial distance between the superior limiting sulcus of the insula and the lateral surface of the pyramidal pathway.

- D12: distance measured at the caudal end of the insula, the shortest lateromedial distance between the intersection of the superior and inferior limiting sulci of the insula and the lateral surface of the IFOF.

\section{Illustrative Cases}

\section{Case 1: Left Frontotemporoinsular Anaplastic Astrocytoma}

Figure 2 shows an MR image obtained in a right-handed patient who had an isolated tonic-clonic generalized seizure 1 month before admission. No preoperative neurological deficit was observed. MRI revealed an image characteristic of a low-grade glioma infiltrating the left insula, temporal operculum, frontal operculum, and frontal orbital area (Yaşargil Type 5A; ${ }^{44}$ Berger-Sanai Classes I, III, and IV ${ }^{32}$ ). DTI tractography revealed that the uncinate fasciculus had an abnormal trajectory as it crossed the superior isthmus but not the anterior isthmus. Interestingly, DTI tractography failed to reconstruct the IFOF, probably due to tumor infiltration and compression. Intraoperative electrical stimulation (IES) of the frontal operculum elicited speech arrest (at the ventral premotor cortex), anomia (at the pars opercularis), and semantic paraphasia (at the pars triangularis). Subcortical electrical stimulation failed to identify eloquent structures at the margins of the surgical cavity, so the resection was extended to the deep tumor margin at the lateral surface of the striatum. The final histopathological diagnosis was anaplastic astrocytoma. The patient presented mild postoperative dysphasia that completely recovered 1 week after surgery.

\section{Case 2: Left Frontotemporoinsular WHO Grade II Astrocytoma}

Figures 3, 4, and 5 show MR images and intraoperative photographs obtained in a right-handed patient who had 5 partial seizures with dysphasia before admission. No preoperative neurological deficit was observed. MRI revealed a left frontotemporoinsular lesion with characteristics of a low-grade glioma (Yaşargil Type 5B; Berger-Sanai Classes I, II, III, and IV). DTI tractography revealed that the uncinate fasciculus and IFOF were medially displaced at the anterior, superior, and temporal isthmi due to tumor compression. IES at the deep portion of the temporal stem induced anomia, corresponding to the IFOF. The final pathology was WHO Grade II astrocytoma. Postoperatively the patient had dysphasia that required cognitive rehabilitation and completely resolved by 3 months after surgery.

\section{Discussion}

The current study revealed that insulo-opercular gliomas cause important distortions of the normal subcortical anatomy of the insular region. In particular, the tumor seems to grow in the space between the IFOF and uncinate fasciculus and the insular convexity, displacing and compressing medially these 2 eloquent bundles. Moreover, these tracts may be completely infiltrated by the tumor, with a total disruption of the bundles. Numerous publications had analyzed the trajectory and relationships of these tracts within the insula. ${ }^{13,14,18,26,37,39,42}$ The 2 tracts are critical landmarks in the insular region, as they separate structures that pass superficial (auditory radiations, claustro-opercular and insulo-opercular fibers of the external and extreme capsules) and deep (the anterior commissure, subcallosal fasciculus, pyramidal pathway and accumbofrontal fasciculus) to them. 
TABLE 1. Demographic, clinical, radiological, and histological characteristics in 22 cases of insular glioma*

\begin{tabular}{|c|c|c|c|c|}
\hline Characteristic & Total $(n=22)$ & EOR < $80 \%(n=11)$ & $E O R \geq 80 \%(n=11)$ & p Value $†$ \\
\hline Patient age (yrs) & & & & 0.61 \\
\hline Mean & 43.6 & 43.2 & 44 & \\
\hline Range & $22.1-66.4$ & $35.5-58.9$ & $22.1-66.4$ & \\
\hline Tumor side & & & & 1.00 \\
\hline Right & $6(27.3)$ & $3(27.3)$ & $3(27.3)$ & \\
\hline Left & $16(72.7)$ & $8(72.7)$ & $8(72.7)$ & \\
\hline Berger-Sanai class & & & & 0.87 \\
\hline I & $21(95.5)$ & $11(100)$ & $10(90.9)$ & \\
\hline II & $14(63.6)$ & $8(72.7)$ & $6(54.5)$ & \\
\hline III & $19(86.4)$ & 9 (81.8) & $10(90.9)$ & \\
\hline IV & $21(95.5)$ & $10(90.9)$ & $11(100)$ & \\
\hline Yaşargil type & & & & 0.88 \\
\hline $3 A$ & $3(13.6)$ & $2(18.2)$ & $1(9.1)$ & \\
\hline $3 B$ & $2(9.1)$ & $1(9.1)$ & $1(9.1)$ & \\
\hline $5 A$ & $9(40.9)$ & $4(36.4)$ & $5(45.5)$ & \\
\hline $5 B$ & $8(36.4)$ & $4(36.4)$ & $4(36.4)$ & \\
\hline Preop tumor volume $\left(\mathrm{cm}^{3}\right)$ & & & & 0.61 \\
\hline Mean & 85.9 & 84 & 87.9 & \\
\hline Range & $25.5-173.8$ & $25.5-173.8$ & $63.4-128.7$ & \\
\hline Histology (\%) & & & & 0.27 \\
\hline WHO Grade II glioma & $15(68.2)$ & $8(72.7)$ & $7(63.6)$ & \\
\hline Anaplastic glioma & $7(31.8)$ & $3(27.3)$ & $4(36.4)$ & \\
\hline IFOF identification (\%) & & & & 0.016 \\
\hline Yes & $14(63.6)$ & $10(90.9)$ & $4(36.4)$ & \\
\hline No & $8(36.4)$ & $1(9.1)$ & $7(63.6)$ & \\
\hline Uncinate identification (\%) & & & & 0.40 \\
\hline Yes & $13(59.1)$ & $6(54.5)$ & $7(63.6)$ & \\
\hline No & $9(40.9)$ & $5(45.5)$ & $4(36.4)$ & \\
\hline
\end{tabular}

EOR = extent of resection; IFOF identification = preoperative identification of IFOF with DTI tractography; uncinate identification = preoperative identification of the uncinate fasciculus with DTI tractography; Yaşargil = Yaşargil classification of insular gliomas.

${ }^{*}$ Values represent numbers of cases (\%) unless otherwise indicated.

$\dagger$ The $p$ values are derived from multivariate logistic regression analysis, comparing the extent of tumor resection and qualitative and quantitative variables. Bold type represents statistical significance.

The IFOF is a long white-matter bundle that directly connects the frontal lobe (orbitofrontal cortex and the prefrontal region) with the posterolateral temporal and occipital lobes. ${ }^{7,21,33}$ The functions of this fascicle are poorly understood, and it seems to be implicated in attention, visual processing, and language. , $^{6-12,15}$ The uncinate fasciculus is a ventral associative C-shaped bundle that connects the anterior temporal lobe with the frontal cortex. ${ }^{7}$ The uncinate fasciculus is considered to belong to the limbic system, and it has been implicated in emotion and behavioral processing, memory, social cognition, and language. ${ }^{1,16}$

\section{Tract Identification and Extent of Insular Glioma Resection}

Tumoral infiltration in low-grade gliomas is usually associated with a decrease in anisotropy values, increasing the probability of a false negative result with DTI tractography (negative tractography results when the real fi-
TABLE 2. Tracts identified in 22 tumoral hemispheres and 20 nontumoral hemispheres

\begin{tabular}{|c|c|c|c|}
\hline \multirow[b]{2}{*}{ Characteristic } & \multicolumn{2}{|c|}{ No. of Hemispheres (\%) } & \multirow[b]{2}{*}{$\begin{array}{c}p \\
\text { Value* }\end{array}$} \\
\hline & $\begin{array}{c}\text { Nontumoral } \\
\text { Hemisphere } \\
\quad(n=20)\end{array}$ & $\begin{array}{c}\text { Tumoral } \\
\text { Hemisphere } \\
(n=22)\end{array}$ & \\
\hline IFOF identification & $20(100)$ & $14(63.6)$ & 0.004 \\
\hline IFOF crosses anterior isthmus & $20(100)$ & $14(100)$ & $>0.05$ \\
\hline IFOF crosses superior isthmus & $20(100)$ & $8(57.1)$ & 0.002 \\
\hline IFOF crosses inferior isthmus & $20(100)$ & $14(100)$ & $>0.05$ \\
\hline UF identification & $20(100)$ & $13(59.1)$ & 0.001 \\
\hline UF crosses anterior isthmus & $20(100)$ & $11(84.6)$ & $>0.05$ \\
\hline UF crosses superior isthmus & $8(40)$ & $4(30.8)$ & $>0.05$ \\
\hline UF crosses inferior isthmus & $20(100)$ & $13(100)$ & $>0.05$ \\
\hline
\end{tabular}

$\mathrm{UF}=$ uncinate fasciculus.

* Based on Fisher exact test. Bold type indicates statistical significance. 
TABLE 3. Distances measured at the insula in 22 patients

\begin{tabular}{cccc}
\hline & \multicolumn{2}{c}{ Mean Value in mm (range) } & \\
\cline { 2 - 3 } $\begin{array}{c}\text { Distance } \\
\text { (mm) }\end{array}$ & $\begin{array}{c}\text { Nontumoral } \\
\text { Hemisphere } \\
(\mathrm{n}=20)\end{array}$ & $\begin{array}{c}\text { Tumoral } \\
\text { Hemisphere } \\
(\mathrm{n}=22)\end{array}$ & p Value \\
\hline D1 & $3.9(1.9-7.7)$ & $10.3(2-28.3)$ & $\mathbf{0 . 0 0 0 2}$ \\
\hline D2 & $8.1(5.0-13.6)$ & $6.4(1.7-18.5)$ & $\mathbf{0 . 0 0 7}$ \\
\hline D3 & $3.9(2.0-5.9)$ & $10.6(2.6-23.3)$ & $\mathbf{0 . 0 1}$ \\
\hline D4 & $7.8(5.3-12.0)$ & $7.2(3.1-13.4)$ & $\mathbf{0 . 0 3}$ \\
\hline D5 & $3.1(1.0-5.0)$ & $7.3(2.0-20.5)$ & $\mathbf{0 . 0 0 0 1}$ \\
\hline D6 & $7.6(6.0-9.0)$ & $7(2.2-14.4)$ & $>0.05$ \\
\hline D7 & $7.1(3.0-11.4)$ & $13.1(4.4-27.5)$ & $\mathbf{0 . 0 0 0 6}$ \\
\hline D8 & $9.1(4.2-14.0)$ & $7.5(3.1-12.2)$ & $\mathbf{0 . 0 3}$ \\
\hline D9 & $11.0(8.0-13.6)$ & $25.3(12.2-47.1)$ & $\mathbf{0 . 0 0 0 1}$ \\
\hline D10 & $0.0(0.0-0.0)$ & $0.0(0.0-0.0)$ & $>0.05$ \\
\hline D11 & $7.1(4.0-10.0)$ & $10.1(4.1-22.7)$ & $>0.05$ \\
\hline D12 & $3.3(3.0-4.0)$ & $7.1(2-15.5)$ & $>0.05$ \\
\hline
\end{tabular}

D1 = shortest lateromedial distance between anterior limiting sulcus of insula and lateral surface of IFOF and UF; D2 = distance measured at anterior isthmus, shortest lateromedial distance between ventricle and lateral surface of IFOF and UF; D3 = shortest lateromedial distance between superior limiting sulcus of insula and lateral surface of IFOF and UF; D4 = distance measured at superior isthmus, shortest lateromedial distance between ventricle and lateral surface of IFOF and UF; D5 = shortest superoinferior distance between inferior limiting sulcus of insula and superior surface of IFOF and UF; D6 = distance measured at temporal stem, shortest superoinferior distance between ventricle and superior surface of IFOF and UF; D7 = shortest lateromedial distance between limen insulae and lateral surface of IFOF and UF; D8 = shortest distance between lateral margin of anterior perforated substance and lateral surface of IFOF and UF; D9 = shortest lateromedial distance between insular apex and lateral surface of IFOF and UF; D10 = shortest lateromedial distance between lateral surface of putamen and medial surface of IFOF and UF; D11 $=$ shortest lateromedial distance between superior limiting sulcus of insula and lateral surface of pyramidal pathway; D12 = distance measured at caudal end of insula, shortest lateromedial distance between intersection of superior and inferior limiting sulci of insula and lateral surface of IFOF.

* Based on Mann-Whitney U-test. Bold type indicates statistical significance.

ber bundle is not interrupted). To improve tractography sensitivity, we used a probabilistic approach, lowering the fractional anisotropy threshold to allow proper reconstruction of the tract when no tract is evident with the usual threshold. ${ }^{2}$ Applying this methodology, we demonstrated that in $36.4 \%$ of insular gliomas the IFOF was infiltrated to the point that DTI tractography failed to reconstruct it. Moreover, in tumoral hemispheres, the identification of the IFOF with DTI tractography was significantly associated to the extent of tumor resection. The multivariate analysis revealed that none of the variables studied (age, tumor side, tumor location, histology, and preoperative tumor volume) were confounders between the DTI results and the extent of resection.

In the group of patients with an extent of resection of at least $80 \%$, the IFOF was identified in only $36.4 \%$ of patients. However, in the patient population presented here, DTI tractography had an excellent positive predictive value of $87.5 \%$; i.e., if the IFOF was not identified preoperatively, the probability of achieving a resection of $80 \%$ or greater was $87.5 \%$. Intraoperative brain mapping stud- ies have recently demonstrated that DTI tractography data are accurate to describe the trajectory of tracts when infiltrated by tumor, as tracking results correlated with those obtained by direct electrical stimulation. ${ }^{2,4}$ Consequently, the complete disruption of the tract may predict the absence of eloquent subcortical areas, increasing the likelihood of a greater resection. In the case presented in Fig. 2 (Case 1), DTI tractography failed to reconstruct the IFOF, probably due to tumor infiltration. Subcortical stimulation failed to identify subcortical functional areas, so resection was extended to the deep tumor margin at the striatum. On the other hand, in the group of patients with an extent of resection less than $80 \%$, the IFOF was identified in $90.9 \%$ of cases. Consequently, in the patient population presented here, DTI tractography had a negative predictive value of $71.4 \%$, which means that if the IFOF was identified preoperatively, the probability of achieving a resection of less than $80 \%$ was $71.4 \%$. Consequently, the presence of the tract in the vicinity of the tumor may predict the identification of subcortical eloquent areas, decreasing the likelihood of a greater resection. In the case presented in Figs. 3-5 (Case 2), DTI tractography was able to reconstruct the IFOF and uncinate fasciculus that were displaced medially due to tumor compression. Subcortical mapping identified the IFOF at the deep portion of the temporal stem, which constituted the deep functional limit with residual tumor below this margin.

In addition to low sensitivity in the case of tumor infiltration, DTI tractography has other inherent shortcomings such as the inaccuracy to map the fiber architecture in areas where different fibers intersect, ${ }^{41}$ or the difficulty in distinguishing the different fascicles in areas where tracts run in parallel (e.g., the optic radiations and the IFOF). ${ }^{5}$ These limitations may have affected the correlation between DTI tractography and fiber dissection.

\section{Transopercular Approach to Insulo-Opercular Gliomas}

The surgical approach to insular gliomas presents the surgeon with specific anatomical, functional, and technical challenges. In fact, in the past, many neurosurgeons were reluctant to attempt tumor removal in the insula due to the high risk of sequelae, and they considered these tumors to be unresectable. A better understanding of the surgical anatomy of this region and recent advances in microsurgical and brain mapping techniques have allowed increased surgical indications for insular gliomas, and have led to improved oncological and functional results. ${ }^{8,17,19,32,34,43}$ The findings established in the present investigation are critical to understanding the subcortical anatomy of the insula when infiltrated by a tumor. In light of this new anatomical data, we will review from a functional perspective the major steps of the surgical approach to insulo-opercular gliomas.

Regarding the selection of the approach to the insula, some authors advocate the systematic use of the transsylvian approach, while others prefer the transopercular approach. ${ }^{8,32,35,44}$ Due to the extensive implementation of intraoperative mapping, more and more surgeons have switched to the transopercular approach to insular gliomas. ${ }^{8,32,35}$ However, the transsylvian approach is the preferred option in insular gliomas without opercular exten- 

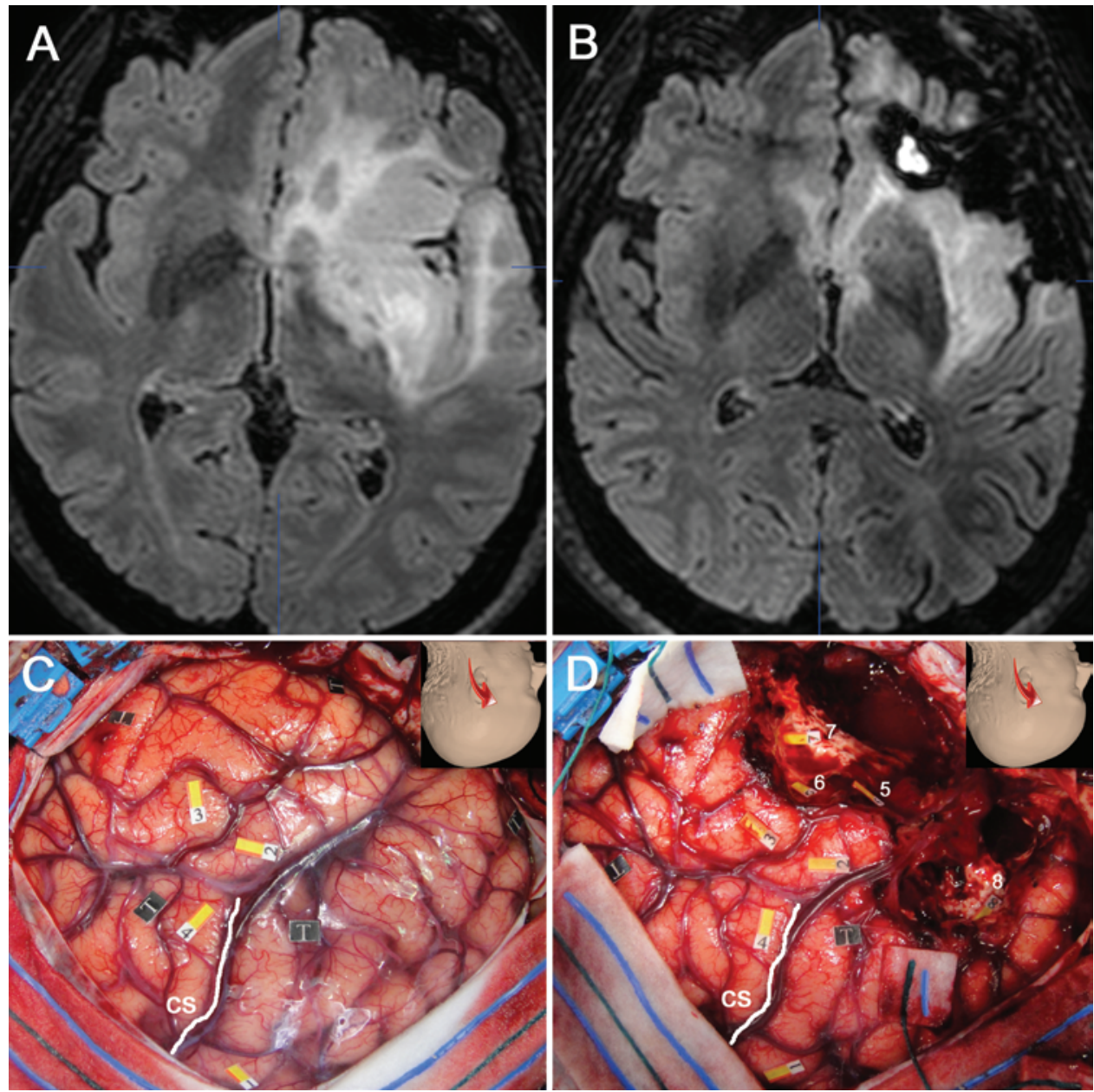

FIG. 5. A: Preoperative axial FLAIR-weighted MRI image of the same case presented in Figs. 3 and 4. The preoperative tumor volume was $133.2 \mathrm{~cm}^{3}$. B: Postoperative axial FLAIR-weighted MR image. The resection was extended until eloquent structures were encountered. The postoperative tumor volume was $34.8 \mathrm{~cm}^{3}$, and the extent of resection was $73.9 \%$. C: Intraoperative photograph taken before tumor resection, where the frontal and temporal lobes and the sylvian fissure are exposed. IES of the cortex elicited the following responses: flag with number $1=$ speech arrest at the ventral premotor cortex, flag with number $2=$ anomia at the posterior part of the superior temporal gyrus, flag with number $3=$ anomia at the posterior part of the middle temporal gyrus, and flag with number $4=$ anarthria at the ventral part of the retrocentral gyrus. CS = central sulcus. D: Intraoperative photograph taken after tumor resection. IES of the white matter elicited the following responses: flags with number 5 and $6=$ anomia, flag with number $7=$ anomia at the IFOF at the temporal stem, and flag with number $8=$ perseveration at the head of the caudate nucleus. $\mathrm{CS}=$ central sulcus. Figure is available in color online only.

sion. Previous publications have described in detail the steps of the transopercular approach. ${ }^{8,32}$ In the first step, IES is applied to the frontal and/or temporal operculum to distinguish between the regions essential and not essential for function, enabling an elective subpial resection of the operculum..$^{32}$ Once the operculum has been removed, the insular component of the tumor is subsequently approached by passing through the isthmi. Subpial dissection is performed to gently remove the tumoral tissue below the peri-insular sulci. This step is a critical aspect of the procedure because it creates a surgical route from the operculum to the insular region, providing access to the insular portion of the tumor.

The preferred route through the isthmus is selected based on the exact location of eloquent connections. The current study demonstrated that tumor infiltration dis- torts the trajectory of the tract within the isthmus. Thus, the IFOF crossed the superior isthmus in all nontumoral hemispheres and in $57.1 \%$ of tumoral hemispheres. Based on this information, we may anticipate that eloquent tracts will not be encountered at the superior isthmus, and that this anatomical route would thus allow for a safer and more effective resection through this anatomical route.

Previous publications have demonstrated that in insuloopercular gliomas, the isthmi are infiltrated and expanded, as they are the anatomical routes crossed by the tumor to pass from the insular region to the operculi. ${ }^{8,9}$ The present work revealed that in insulo-opercular gliomas, the distance between the peri-insular sulci and the superficial surface of the IFOF and uncinate fasciculus was enlarged while the distance between these tracts and the ventricle was reduced (Figs. 3 and 4). Consequently, the current 
study revealed that tumors pass from the insula to the operculum through the space between the lateral surface of the IFOF and the peri-insular sulci. This space, superficial to the IFOF and uncinate fasciculus, is crossed by the U-fibers that connect the insular cortex and claustrum with the operculi. Therefore, the surgical route from the operculi to the insula through the isthmi should pass through this space, between the lateral surface of the IFOF and uncinate fasciculus and the peri-insular sulci. The current anatomical data are supported by previous IES mapping studies, where the IFOF was identified as the deep limit of the resection within the frontal and temporal isthmi in the dominant hemisphere. ${ }^{8,10,11}$ The current data indicate that this space superficial to the IFOF is very narrow in the nontumoral hemisphere (at the temporal stem: average $3 \mathrm{~mm}$, range 1-5 mm) (Fig. 3). However, in an insuloopercular glioma, the isthmi are enlarged, and the space between the tracts and the peri-insular sulci is widely expanded (at the temporal stem: average $7 \mathrm{~mm}$, range 2-21 mm) (Fig. 3). Interestingly, the preservation at the level of the temporal stem of the deep functional boundary of the IFOF and uncinate fasciculus will also avoid damaging the optic radiations because they are located below this limit. Likewise, at the anterior and superior isthmi, the preservation of these functional tracts will avoid damaging deeper, important structures such as the accumbofrontal fasciculus, subcallosal fasciculus, and the head of the caudate nucleus.

In the next step of the procedure, the insular component of the tumor is removed subpially. As has been previously reported, the resection is performed from a lateral to medial direction until deep eloquent structures are encountered. ${ }^{8,19,32}$ The present work revealed that in insuloopercular gliomas, the distance between the insular apex and the superficial surface of the IFOF and uncinate fasciculus was enlarged. Moreover, these tracts were in direct contact with the lateral surface of the putamen; as in all tumoral hemispheres, there was no space between these tracts and the putamen (Figs. 3 and 4). The present work also revealed that insular gliomas grow in the space between the tracts and the insular surface, compressing the tracts in a medial direction. Consequently, at the anterior and ventral portion of the insula in the dominant hemisphere, the deep functional margin of resection is the IFOF. It is mandatory to use IES with continuous neuropsychological testing to extend the resection until reaching this eloquent limit.

The extent of tumor resection at the level of the limen insulae remains a subject of debate. In insular gliomas surgery, an injury to the lateral lenticulostriate arteries represents the main cause of permanent neurological deficit, leading to permanent hemiparesis due to a deep stroke in the internal capsule. ${ }^{8,19,28,31}$ The present work revealed that in insulo-opercular gliomas, the distance between the limen insulae and the IFOF and uncinate fasciculus was enlarged, while the distance between the lateral margin of the anterior perforated substance and the lateral surface of the tracts was reduced. Consequently, at the limen insulae, gliomas seem to grow in the space between the tracts and the insular surface, compressing the tracts in a medial direction. This is valuable information for surgical planning, as it helps to delineate that in the dominant hemisphere the deep functional margin of resection at the limen insulae will be the IFOF. It is worth noting that the preservation of the deep functional boundary of the IFOF and uncinate fasciculus at the level of the limen insulae will also avoid damaging the lateral lenticulostriate arteries because they are located below this limit.

The final step of the procedure is the approach to the tumor that infiltrates the posterior insula. ${ }^{8,19,32}$ In the present work, the anatomical relationships between the IFOF and the caudal end of the insula were analyzed, and there were no differences between the tumoral and nontumoral hemispheres. However, we cannot drawn firm conclusions on this issue as the tumors were preferentially located in the anterior part of the insula in the present series.

The existing classification methods for insular gliomas are based on growth patterns and tumor location. ${ }^{44} \mathrm{Sa}-$ nai et al. ${ }^{32}$ recently proposed a very useful classification based on tumor location, resectability, and functional outcomes. The current findings emphasize the importance of subcortical anatomy to anticipate the surgical risk and to better assess the feasibility of tumor resection. Therefore, these data should be incorporated in the future classifications of insular gliomas. Further studies are necessary, with a larger cohort of insular gliomas that cover other anatomical locations beyond those described in the present series.

\section{Conclusions}

The data presented in this study revealed that insular gliomas frequently infiltrate and compress the IFOF and uncinate fasciculus, creating distortion of the normal anatomy or complete disruption of the bundles. The tumor displaces and compresses the tracts medially as it grows in the space between the tracts and the insular surface. This information is of major significance for planning of insular glioma surgery and predicting the neurological risks of the procedure, as these connections represent the deep functional margin of resection at the level of the isthmus and insula.

On the other hand, these tracts may be completely infiltrated by the tumor, with a total disruption of the bundles. When this occurs, the probability to identify subcortical eloquent areas is lower, increasing the likelihood of a greater tumor resection. In the current study, if the IFOF was not identified preoperatively, the probability of achieving a resection of $80 \%$ or greater was $87.5 \%$.

\section{References}

1. Agosta F, Henry RG, Migliaccio R, Neuhaus J, Miller BL, Dronkers NF, et al: Language networks in semantic dementia. Brain 133:286-299, 2010

2. Bello L, Gambini A, Castellano A, Carrabba G, Acerbi F, Fava E, et al: Motor and language DTI fiber tracking combined with intraoperative subcortical mapping for surgical removal of gliomas. Neuroimage 39:369-382, 2008

3. Burgess PK, Kulesa PM, Murray JD, Alvord EC Jr: The interaction of growth rates and diffusion coefficients in a threedimensional mathematical model of gliomas. J Neuropathol Exp Neurol 56:704-713, 1997

4. Castellano A, Bello L, Michelozzi C, Gallucci M, Fava E, 
Iadanza A, et al: Role of diffusion tensor magnetic resonance tractography in predicting the extent of resection in glioma surgery. Neuro Oncol 14:192-202, 2012

5. Catani M, Jones DK, Donato R, Ffytche DH: Occipito-temporal connections in the human brain. Brain 126:2093-2107, 2003

6. Catani M, Mesulam M: What is a disconnection syndrome? Cortex 44:911-913, 2008

7. Catani M, Thiebaut de Schotten M: A diffusion tensor imaging tractography atlas for virtual in vivo dissections. Cortex 44:1105-1132, 2008

8. Duffau H: A personal consecutive series of surgically treated 51 cases of insular WHO Grade II glioma: advances and limitations. J Neurosurg 110:696-708, 2009

9. Duffau H, Capelle L: Preferential brain locations of lowgrade gliomas. Cancer 100:2622-2626, 2004

10. Duffau H, Gatignol P, Mandonnet E, Capelle L, Taillandier L: Intraoperative subcortical stimulation mapping of language pathways in a consecutive series of 115 patients with Grade II glioma in the left dominant hemisphere. J Neurosurg 109:461-471, 2008

11. Duffau H, Gatignol P, Mandonnet E, Peruzzi P, TzourioMazoyer N, Capelle L: New insights into the anatomofunctional connectivity of the semantic system: a study using cortico-subcortical electrostimulations. Brain 128:797-810, 2005

12. Epelbaum S, Pinel P, Gaillard R, Delmaire C, Perrin M, Dupont S, et al: Pure alexia as a disconnection syndrome: new diffusion imaging evidence for an old concept. Cortex 44:962-974, 2008

13. Fernandez-Miranda JC, Pathak S, Engh J, Jarbo K, Verstynen $\mathrm{T}$, Yeh FC, et al: High-definition fiber tractography of the human brain: neuroanatomical validation and neurosurgical applications. Neurosurgery 71:430-453, 2012

14. Fernández-Miranda JC, Rhoton AL Jr, Kakizawa Y, Choi C, Alvarez-Linera J: The claustrum and its projection system in the human brain: a microsurgical and tractographic anatomical study. J Neurosurg 108:764-774, 2008

15. Fox CJ, Iaria G, Barton JJ: Disconnection in prosopagnosia and face processing. Cortex 44:996-1009, 2008

16. Fujie S, Namiki C, Nishi H, Yamada M, Miyata J, Sakata $\mathrm{D}$, et al: The role of the uncinate fasciculus in memory and emotional recognition in amnestic mild cognitive impairment. Dement Geriatr Cogn Disord 26:432-439, 2008

17. Hentschel SJ, Lang FF: Surgical resection of intrinsic insular tumors. Neurosurgery 57 (1 Suppl):176-183, 2005

18. Kier EL, Staib LH, Davis LM, Bronen RA: MR imaging of the temporal stem: anatomic dissection tractography of the uncinate fasciculus, inferior occipitofrontal fasciculus, and Meyer's loop of the optic radiation. AJNR Am J Neuroradiol 25:677-691, 2004

19. Lang FF, Olansen NE, DeMonte F, Gokaslan ZL, Holland EC, Kalhorn C, et al: Surgical resection of intrinsic insular tumors: complication avoidance. J Neurosurg 95:638-650, 2001

20. Mandonnet E, Capelle L, Duffau H: Extension of paralimbic low grade gliomas: toward an anatomical classification based on white matter invasion patterns. J Neurooncol 78:179-185, 2006

21. Martino J, Brogna C, Robles SG, Vergani F, Duffau H: Anatomic dissection of the inferior fronto-occipital fasciculus revisited in the lights of brain stimulation data. Cortex 46:691-699, 2010

22. Martino J, da Silva-Freitas R, Caballero H, Marco de Lucas E, García-Porrero JA, Vázquez-Barquero A: Fiber dissection and diffusion tensor imaging tractography study of the temporoparietal fiber intersection area. Neurosurgery 72 (1 Suppl Operative):87-98, 2013
23. Martino J, De Witt Hamer PC, Berger MS, Lawton MT, Arnold CM, de Lucas EM, et al: Analysis of the subcomponents and cortical terminations of the perisylvian superior longitudinal fasciculus: a fiber dissection and DTI tractography study. Brain Struct Funct 218:105-121, 2013

24. Martino J, De Witt Hamer PC, Vergani F, Brogna C, de Lucas EM, Vázquez-Barquero A, et al: Cortex-sparing fiber dissection: an improved method for the study of white matter anatomy in the human brain. J Anat 219:531-541, 2011

25. Martino J, Gomez E, Bilbao JL, Dueñas JC, Vázquez-Barquero A: Cost-utility of maximal safe resection of WHO grade II gliomas within eloquent areas. Acta Neurochir (Wien) 155:41-50, 2013

26. Martino J, Vergani F, Robles SG, Duffau H: New insights into the anatomic dissection of the temporal stem with special emphasis on the inferior fronto-occipital fasciculus: implications in surgical approach to left mesiotemporal and temporoinsular structures. Neurosurgery 66 (3 Suppl Operative): $4-12,2010$

27. Metz-Lutz M, Kremin H, Deloche G, Hannequin D, Ferrand I, Perrier D, et al: Standardisation d'un test de dénomination orale: contrôle des effets de l'àge, du sexe, et du niveau de scolarité chez les sujets adultes normaux. Rev Neuropsychol (Marseille) 1:73-95, 1991

28. Neuloh G, Pechstein U, Schramm J: Motor tract monitoring during insular glioma surgery. J Neurosurg 106:582-592, 2007

29. Nimsky C, Ganslandt O, Cerny S, Hastreiter P, Greiner G, Fahlbusch R: Quantification of, visualization of, and compensation for brain shift using intraoperative magnetic resonance imaging. Neurosurgery 47:1070-1080, 2000

30. Rhoton AL Jr: The cerebrum. Neurosurgery 51 (4 Suppl):S1-S51, 2002

31. Saito R, Kumabe T, Inoue T, Takada S, Yamashita Y, Kanamori M, et al: Magnetic resonance imaging for preoperative identification of the lenticulostriate arteries in insular glioma surgery. Technical note. J Neurosurg 111:278-281, 2009

32. Sanai N, Polley MY, Berger MS: Insular glioma resection: assessment of patient morbidity, survival, and tumor progression. J Neurosurg 112:1-9, 2010

33. Sarubbo S, De Benedictis A, Maldonado IL, Basso G, Duffau $\mathrm{H}$ : Frontal terminations for the inferior fronto-occipital fascicle: anatomical dissection, DTI study and functional considerations on a multi-component bundle. Brain Struct Funct 218:21-37, 2013

34. Simon M, Neuloh G, von Lehe M, Meyer B, Schramm J: Insular gliomas: the case for surgical management. J Neurosurg 110:685-695, 2009

35. Skrap M, Mondani M, Tomasino B, Weis L, Budai R, Pauletto G, et al: Surgery of insular nonenhancing gliomas: volumetric analysis of tumoral resection, clinical outcome, and survival in a consecutive series of 66 cases. Neurosurgery 70:1081-1094, 2012

36. Smith JS, Chang EF, Lamborn KR, Chang SM, Prados MD, Cha $S$, et al: Role of extent of resection in the long-term outcome of low-grade hemispheric gliomas. J Clin Oncol 26:1338-1345, 2008

37. Straus D, Byrne RW, Sani S, Serici A, Moftakhar R: Microsurgical anatomy of the transsylvian translimen insula approach to the mediobasal temporal lobe: Technical considerations and case illustration. Surg Neurol Int 4:159, 2013

38. Swanson KR, Bridge C, Murray JD, Alvord EC Jr: Virtual and real brain tumors: using mathematical modeling to quantify glioma growth and invasion. J Neurol Sci 216:1-10, 2003 
39. Tanriover N, Rhoton AL Jr, Kawashima M, Ulm AJ, Yasuda A: Microsurgical anatomy of the insula and the sylvian fissure. J Neurosurg 100:891-922, 2004

40. Vanaclocha V, Sáiz-Sapena N, García-Casasola C: Surgical treatment of insular gliomas. Acta Neurochir (Wien) 139:1126-1135, 1997

41. Wang F, Sun T, Li XG, Liu NJ: Diffusion tensor tractography of the temporal stem on the inferior limiting sulcus. J Neurosurg 108:775-781, 2008

42. Wen HT, Rhoton AL Jr, de Oliveira E, Castro LH, Figueiredo EG, Teixeira MJ: Microsurgical anatomy of the temporal lobe: part 2-sylvian fissure region and its clinical application. Neurosurgery 65 (6 Suppl):1-36, 2009

43. Wu AS, Witgert ME, Lang FF, Xiao L, Bekele BN, Meyers $\mathrm{CA}$, et al: Neurocognitive function before and after surgery for insular gliomas. J Neurosurg 115:1115-1125, 2011

44. Yaşargil MG, Reeves JD: Tumours of the limbic and paralimbic system. Acta Neurochir (Wien) 116:147-149, 1992

\section{Author Contributions}

Conception and design: Martino. Acquisition of data: Martino, Mato, Marco de Lucas, García-Porrero, Gabarrós, FernándezCoello. Analysis and interpretation of data: Martino. Drafting the article: Martino. Critically revising the article: Martino, Mato, Marco de Lucas, García-Porrero, Gabarrós, Fernández-Coello. Reviewed submitted version of manuscript: all authors. Approved the final version of the manuscript on behalf of all authors: Martino. Statistical analysis: Martino. Administrative/technical/material support: Martino. Study supervision: Martino.

\section{Correspondence}

Juan Martino, Department of Neurological Surgery, Hospital Universitario Marqués de Valdecilla and Fundación Instituto de Investigación Marqués de Valdecilla (IDIVAL), Avda. Valdecilla s/n, Santander, Cantabria 39008, Spain. email: juan.martino@ hotmail.com. 